Revue d'histoire de l'Amérique française

75. REVUE D.HISTOIRE DE L'AMÉRIQUE FRANÇAISE

\title{
Présentation des papiers contrecoeur
}

\section{Fernand Grenier}

Volume 6, numéro 2, septembre 1952

URI : https://id.erudit.org/iderudit/301519ar

DOI : https://doi.org/10.7202/301519ar

Aller au sommaire du numéro

Éditeur(s)

Institut d'histoire de l'Amérique française

ISSN

0035-2357 (imprimé)

1492-1383 (numérique)

Découvrir la revue

Citer cet article

Grenier, F. (1952). Présentation des papiers contrecoeur. Revue d'histoire de l'Amérique française, 6(2), 247-251. https://doi.org/10.7202/301519ar d'utilisation que vous pouvez consulter en ligne.

https://apropos.erudit.org/fr/usagers/politique-dutilisation/ 


\section{PRÉSENTATION DES PAPIERS CONTRECOEUR*}

Le Séminaire de Québec possède l'un des plus importants dépôts d'archives de l'Amérique du Nord. Plusieurs historiens canadiens, américains et français, ont d'ailleurs déjà utilisé quelquesuns des nombreux documents que recèlent ces riches archives. Avec cette édition des Papiers Contrecaur et autres documents concernant le conflit anglo-français sur l'Ohio de 1745 à $1756^{* *}$ les Archives du Séminaire de Québec entreprennent donc une série de publications qui devraient rendre de précieux services à l'histoire canadienne et à l'histoire américaine en général.

Le fonds Viger-Verreau constitue certainement l'un des plus variés et des plus intéressants des Archives du Séminaire de Québec. Il se compose d'une centaine de boîtes et cartons remplis de documents - manuscrits pour la plupart - placés dans des chemises ou simplement groupés en liasses. On doit y ajouter aussi plusieurs grands cahiers contenant des documents de toutes sortes.

Le fonds Viger-Verreau tire son nom de Jacques Viger $^{1}$ et de l'abbé Hospice-Anthelme Verreau ${ }^{2}$ qui tous les deux s'intéressèrent

* Etude présentée à la réunion générale de l'Institut d'histoire de l'Amérique française, à Québec, le 17 avril 1952.

** Paraîtra cet automne, aux Presses Universitaires Laval, 1952.

1. Jacques Viger, né à Montréal le 7 mai 1787; rédacteur au journal Le Canadien de Québec en 1808-1809; capitaine des Voltigeurs canadiens durant la guerre de 1812; arpenteur et inspecteur des routes et ponts de Montréal en 1813; chargé du recensement de Montréal en 1825 et premier maire de cette ville de 1833 à 1839; après les troubles de 1837-1838, il eut à s'occuper du recouvrement des pertes subies par les habitants; décédé le 12 décembre 1858. Historiographe, collectionneur d'archives et fondateur de la Société historique de Montréal. Avait épousé, en 1808, Marie-Marguerite de La Corne de Chapt de Saint-Luc, veuve du lieutenant John Lennox. Sur Viger, voir l'abbé Camille Roy, "Jacques Viger", le Bulletin du Parler français au Canada (1909-1910):42-55; E.-Z. Massicotte, "Jacques Viger et sa famille", le Bulletin des Recherches historiques, (1915): 148-149.

2. L'abbé Hospice-Anthelme-Jean-Baptiste Verreau (1828-1901), éducateur, principal de l'Ecole Normale Jacques-Cartier de Montréal, président de la Société historique de Montréal. En 1873, le gouvernement canadien l'envoya à Londres et 
à l'histoire canadienne, écrivirent quelques livres et collectionnèrent de nombreuses pièces d'archives qu'ils découvraient un peu partout dans les familles et les institutions de Montréal, de Québec ou d'ailleurs. Mémoires, lettres, brochures, plans, cartes et gravures: on trouve de tout dans ces collections. La très grande partie des documents sont des originaux; on a cependant un certain nombre de copies très soignées dont, en plusieurs cas, nous avons pu vérifier l'exactitude ${ }^{3}$. L'abbé Verreau avait déjà fait l'acquisition des papiers de Jacques Viger; à sa mort, il légua ses précieux documents au Séminaire de Québec dont il était un ancien élève. Les pièces manuscrites furent laissées aux Archives du Séminaire tandis que l'imposante collection de Canadiana de l'abbé Verreau était déposée à la Bibliothèque générale de l'Université Laval où elle figure encore avec honneur.

Les documents du fonds Viger-Verreau se rapportent à presque toutes les questions importantes de l'histoire canadienne, depuis les origines jusque vers la fin du dix-neuvième siècle. C'est dans ce fonds que se trouvent les Papiers Contrecœur, Marin, Le Gardeur de Saint-Pierre et de Beaujeu qui concernent l'occupation française de la vallée de l'Ohio. Plusieurs autres documents du fonds VigerVerreau intéressent de façon plus générale les origines de la guerre de Sept ans en Amérique. Mentionnons les Papiers de Gaspé, Lacorne, Lacorne Saint-Luc et Lacorne Du Breuil, la Perrière, Demuy, de Boucherville, une série de documents sur Braddock et une collection de lettres de Contrecœur, Vaudreuil, Dumas, Desligneris et La Valtrie copiées par Jacques Viger. Tous ces documents, à cause de leur intérêt, mériteraient d'être édités car ils éclairent quelques aspects ignorés mais importants des origines de cette guerre qui derait modifier si profondément le sort de l'Amérique française.

à Paris préparer un inventaire des archives intéressant le Canada. Voir l'abbé Armand Yon, L'abbe H.-A. Verreau, (Fides, Montréal, 1946, 208p., un portrait hors texte). L'abbé Yon n'a pas utilisé les documents du fonds Viger-Verreau des Archives du Séminaire de Québec et cette biographie est loin d'être définitive.

3. Jacques Viger a laissé 44 cahiers manuscrits intitulés $M a$ Saberdache et 5 autres intitulés Opuscules. Plusieurs des documents copiés sont inédits et mériteraient d'être publiés.

4. On trouvera une description plus détaillée de ces collections dans la Bibliographie. 
Dans la présente édition, nous nous en sommes tenu à la question de l'Ohio et les documents édités ont été choisis dans les Papiers Contrecœur, Marin, Le Gardeur de Saint-Pierre et de Beaujeu ${ }^{5}$.

Claude-Pierre Pécaudy de Contrecour fut l'une des plus importantes figures canadiennes de la fin du régime français ${ }^{6}$. En 1752, il était commandant à Niagara, et, à ce titre, devait s'occuper des portages de Niagara, des conditions générales de la traite dans cette région, de l'approvisionnement des forts environnants et des relations avec les Indiens. En 1753, sa principale n:ission fut d'assurer le succès de l'expédition que le gouverneur Duquesne envoyait pour occuper la vallée de l'Ohio et y construire des forts. L'état des portages de Niagara devait être excellent et il appartenait aux barques de Niagara d'assurer le transport des soldats, des ouvriers et aussi de toutes les provisions nécessaires. En 1754, Contrecœur était commandant de tous les postes de la Belle-Rivière; il eut alors à déloger les Anglais qui avaient commencé à s'établir au confluent de la Monongahéla et de l'Ohio; sur ce même site, il dirigea la construction de l'important fort Duquesne. Contrecœur dut alors s'opposer aux Anglais, traiteurs et militaires, fut mêlé à l'affaire Jumonville, dut s'occuper des prisonniers anglais ainsi que des relations souvent difficiles avec les Indiens de la Belle-Rivière. Il était encore commandant au fort Duquesne lorsque Braddock vint attaquer les établissements français de l'Ohio, au début de juillet 1755 . Epuisé et malade, Contrecœur revint à Montréal en 1756. Cette même année, il recevait la croix de Saint-Louis en reconnaissance des nombreux services qu'il avait rendus à la Nouvelle-France. Trois ans plus tard, il vivait retiré avec une pension annuelle de 540 livres. Après la conquête, Claude-Pierre Pécaudy de Contrecœur passait pour l'un des Canadiens les plus influents ${ }^{7}$. Le 17 août 1775, il était assermenté comme membre du premier Conseil législatif; il ne put cependant assister qu'à une seule séance puisqu'i! décédait, à Montréal, le 13 décembre suivant.

5. Les documents non publiés dans cette édition le seront probablement dans un second volume.

6. Sur Contrecœur, voir F.-J. Audet, Contrecœur (Montréal, 1940) et l'abbé Auguste Gosselin, Une famille de heros, les Pécaudy de Contreceeur (extrait de la Revue Catholique de Normandie, Evreux, 1904).

7. Carleton à Hillsborough, le 15 mars 1769, $\mathrm{AC}$, série $\mathrm{Q}$, vol. 6. 
Les Papiers Contrecœur forment la plus grande partie des documents publiés dans cette édition. Parmi les signataires des lettres adressées à Contrecœur figurent les noms de Duquesne, Bigot, Varin, Marin, Péan, La Chauvignerie, Saint-Blin, La Perrière, Benoist, d'une dizaine d'autres officiers ou traiteurs et aussi de quelques membres de la famille de Contrecœur. Une vingtaine de brouillons de lettres écrites par Contrecœur sont également incluses. Enfin divers documents font aussi partie des Papiers Contrecœur tels un important Journal de Georges Washington (1754), le Journal de la campagne de M. de Villiers au fort Nécessité (1754), des comptes rendus de conférences avec les Tsonnontouans, les Loups, les Chaouanons et autres Indiens de la Belle-Rivière, deux cahiers de dépenses pour la construction du fort Duquesne, les listes de provisions nécessaires dans les forts, des dépositions de déserteurs anglais ${ }^{8}$.

En 1753, le gouverneur Duquesne avait confié à Pierre-Paul de la Malgue, sieur de Marin, le commandement d'une expédition de 2,300 hommes, soldats et ouvriers, qui devaient assurer la prise de possession de l'Ohio et y construire les forts de la Presqu'île et de la Rivière-au-Bœuf. Michel-Jean-Hugues Péan commandait en second lors de cette même expédition. Les Papiers Marin pour l'année 1753 sont publiés dans cette édition; au nombre d'une vingtaine, ils comprennent des lettres de Duquesne, Dumas, Joncaire, Baby et aussi deux brouillons de lettres de Marin à Joncaire et Dumas. Ces Papiers renferment d'intéressants détails sur les difficultés rencontrées par l'expédition, sur les précautions à prendre à l'égard des Indiens, sur la construction des forts de la Presqu'île et de la Rivière-au-Bœuf et sur la maladie qui s'introduisit dans les troupes et devait finalement emporter le commandant lui-même le 29 octobre $1753^{9}$.

Jacques Le Gardeur de Saint-Pierre remplaça Marin après la mort de ce dernier. Pendant les quelques mois qu'il commanda au fort de la Rivière-au-Bœuf, Le Gardeur de Saint-Pierre reçut Georges

8. On trouvera dans la Liste des documents l'énumération détaillée de tous les Papiers Contrecour et autres.

9. Sur Marin, voir Régis Roy, "Paul Marin de la Malgue", Mémoires de la Societé Royale du Canada (1904), section I: 27-30. 
Washington, ambassadeur détaché par le gouverneur Robert Dinwiddie de la Virginie pour aller sommer les Français de se retirer des terres de la Belle-Rivière. La fière réponse du commandant français se trouve parmi les Papiers Le Gardeur de Saint-Pierre que nous avons trouvés dans le fonds Viger-Verreau.

Au cours de 1755, l'événement important du côté de la BelleRivière fut assurément la bataille de la Monongahéla au cours de laquelle le commandant français, Daniel-Hyacinthe-Marie de Beaujeu, perdit glorieusement la vie. Quelques Papiers de Beaujeu passèrent apparemment aux mains de Contrecœur et font maintenant partie du fonds Viger-Verreau. Dix de ces documents forment la liste des troupes de milice levées dans les gouvernements de Montréal, Trois-Rivières et Québec pour constituer le détachement de la Belle-Rivière, au printemps de 1755. La publication de ces pièces devrait intéresser les généalogistes canadiens.

La publication de cette importante série de documents a été rendue possible grâce à la collaboration de la Pennsylvania Historical and Museum Commission, des Archives du Séminaire de Québec, de l'Institut d'histoire et de géographie de l'Université Laval et des Presses Universitaires Laval. Nous croyons fermement que l'on devrait porter plus d'attention à la publication de pièces d'archives susceptibles d'intéresser l'histoire de notre pays. Nos grands hommes politiques, nos littérateurs, des personnages éminents du monde religieux n'ont pas encore été édités. On devrait s'adonner à ce travail le plus tôt possible. Pour nous, nous espérons seulement que cette édition saura rendre quelques services à l'histoire canadienne.

Fernand Grenier, L. ès L., professeur au Petit Séminaire de Quebec et à l'Université Laval. 